\title{
Pneumonia bacteriana em jabuti-piranga (Chelonoidis carbonaria): aspectos clínicos, microbiológicos, radiológicos e terapêutica ${ }^{1}$
}

\author{
Marcelo M. Silveira², Thais O. Morgado ${ }^{3}$, Érika R. Lopes ${ }^{4}$, Guilherme V. Kempe ${ }^{3}$, \\ Sandra H.R. Correa ${ }^{3}$, Isabela de Godoy ${ }^{2}$, Luciano Nakazato ${ }^{2}$ e Valéria Dutra ${ }^{2 *}$
}

\begin{abstract}
Silveira M.M., Morgado T.O., Lopes E.R., Kempe G.V., Correa S.H.R., Godoy I., Nakazato L. \& Dutra V. 2014. [Bacterial pneumonia in red-footed tortoise (Chelonoidis carbonaria): Clinical aspects, microbiological, radiological and therapeutic.] Pneumonia bacteriana em jabuti-piranga (Chelonoidis carbonaria): aspectos clínicos, microbiológicos, radiológicos e terapêutica. Pesquisa Veterinária Brasileira 34(9):891-895. Departamento de Clínica Médica Veterinária, Faculdade de Agronomia, Medicina Veterinária e Zootecnia, Universidade Federal de Mato Grosso, Av. Fernando Corrêa da Costa s/n, Coxipó, Cuiabá, MT 78068-900, Brazil. E-mail: valdutra@ufmt.br

Pneumonia is a common respiratory disease in clinical of reptiles. Infectious agents are capable of causing primary pneumonia in reptiles maintained in captivity, but in most cases are secondary to problems of management, hygiene and nutrition. The aim of this study was to report the occurrence of bacterial pneumonia in red-footed tortoise (Chelonoidis carbonaria), and describe the clinical, microbiologic, radiographic and therapeutic management. The animal showed signs of respiratory disorders and has been described in the clinical history before diagnosis of pneumonia. The radiographic findings were suggestive of pneumonia/pulmonary edema. Based on the displayed radiographic examination and clinical signs began treatment with administration of chloramphenicol (40 mg/ $\mathrm{kg} / \mathrm{SID} / \mathrm{IM}$ ) for ten days. Were isolated Klebsiella spp. and Citrobacter spp. bacterial culture done collecting endotracheal lavage. Both with multiple antibiotic resistance profile tested. Treatment protocol was instituted using gentamicin $(5 \mathrm{mg} / \mathrm{kg} / \mathrm{IM})$ applications into seven intervals of $72 \mathrm{~h}$. There was improvement in clinical signs of the animal, but the presence of nasal secretion was still observed. New radiographic examination, demonstrating slight decrease in the opacity of the right lung field and no significant change in the left lung field in craniocaudal projection was performed. Because of the persistence of clinical signs presented new collection endotracheal material was performed, and there was isolation of Citrobacter spp. and Enterobacter spp. From the results obtained in the antibiogram, was instituted new protocol with the use of amikacin $(2.5 \mathrm{mg} / \mathrm{kg} / \mathrm{IM})$ applications into seven intervals of $72 \mathrm{~h}$. After antibiotic therapy, other radiological examination was performed, and showed satisfactory reduction in pulmonary function and clinical signs.
\end{abstract}

INDEX TERMS: Reptilia, Testudinidae, Enterobacteriaceae, pneumonia, wild animals, hypovitaminosis A.

\footnotetext{
${ }^{1}$ Recebido em 8 de janeiro de 2014.

Aceito para publicação 3 de setembro 2014.

${ }^{2}$ Laboratório de Microbiologia Veterinária e Biologia Molecular Veterinária, Universidade Federal de Mato Grosso (UFMT), Av. Fernando Corrêa s/n, Coxipó, Cuiabá, MT 78060-900, Brasil. *Autor para correspondência: valdutra@ufmt.br

${ }^{3}$ Clínica de Animais Silvestres, Hospital Veterinário UFMT, Av. Fernando Corrêa da Costa s/n, Coxipó, Cuiabá, MT.

${ }^{4}$ Serviço de Diagnóstico por Imagem, Hospital Veterinário UFMT, Av. Fernando Corrêa da Costa s/n, Coxipó, Cuiabá, MT.
}

RESUMO.- A pneumonia é uma doença respiratória comum na clínica de répteis. Agentes infecciosos são capazes de causar pneumonia primária em répteis mantidos em cativeiro, porém na maioria dos casos, são secundárias a problemas de manejo, higiene e nutricionais. 0 objetivo desse trabalho foi relatar a ocorrência de pneumonia bacteriana em jabuti-piranga (Chelonoidis carbonaria), e descrever o diagnóstico clínico, microbiológico, radiográfico e a conduta terapêutica. $\mathrm{O}$ animal apresentava sinais de distúr- 
bios respiratórios e foi descrito durante a anamnese que houve um diagnostico anterior de pneumonia. Os achados radiográficos foram sugestivos de pneumonia/edema pulmonar. Baseado nos exames radiográficos e sinais clínicos apresentados iniciou-se o tratamento com administração de Cloranfenicol (40mg/kg/SID/IM) por 10 dias. Foram isoladas Klebsiella spp. e Citrobacter spp. da cultura bacteriana realizada da coleta de lavado endotraqueal. Ambas com perfil de resistência múltipla aos antibióticos testados. Instituiu-se protocolo terapêutico utilizando Gentamicina (5mg/kg/IM), em sete aplicações com intervalos de $72 \mathrm{~h}$. Após o segundo protocolo terapêutico notou-se melhora dos sinais clínicos do animal, porém foi observada a persistência de secreção nasal. Foi realizado novo exame radiográfico, demonstrando discreta diminuição na opacidade do campo pulmonar direito e nenhuma alteração significativa no campo pulmonar esquerdo na projeção craniocaudal. Devido à permanência do sinal clínico apresentado, nova coleta de material endotraqueal foi realizada, e houve isolamento de Citrobacter spp. e Enterobacter spp. A partir dos resultados obtidos no antibiograma, instituiu-se novo protocolo com uso de amicacina $(2,5 \mathrm{mg} / \mathrm{kg} / \mathrm{IM})$, em sete aplicações com intervalos de 72h. Após antibioticoterapia, outro exame radiológico foi realizado, e demonstrou redução satisfatória do quadro pulmonar, e sinais clínicos.

TERMOS DE INDEXAÇÃO: Reptilia, Testudinidae, Enterobacteriaceae, pneumonia, animais silvestres, hipovitaminose A.

\section{INTRODUÇÃO}

As maiores taxas de morbidade e mortalidade encontradas em répteis são causadas pelas doenças infecciosas. Muitas dessas doenças podem ser tratadas com sucesso se reconhecidas ha tempo, sendo quase sempre secundárias a imunossupressão, e frequentemente associadas com o estresse de cativeiro. As doenças bacterianas são comuns e, como a maioria das infecções causadas por agentes oportunistas é necessária uma abordagem ampla para garantir o sucesso de um plano terapêutico (Mader 2006).

A pneumonia bacteriana é uma doença respiratória de apresentação comum na clinica de répteis. Agentes infecciosos são capazes de causar pneumonia primária em espécies mantidas em cativeiro, porém na maioria dos casos, são secundários a problemas de manejo, higiene e nutricionais (Goldstein et al. 1981, Mader 2006, Jacobson 2007, Benites et al. 2013). O diagnóstico clínico de pneumonia deve ser baseado em sinais clínicos, histórico de terapia adotada, exame físico, seguido por técnicas auxiliares para o diagnóstico (Mader 2006). Sinais como observar a presença da boca aberta durante a respiração, corrimento nasal e dispneia são comuns. Vários micro-organismos podem causar doenças do trato respiratório, entre eles Aeromonas spp. e Pseudomonas spp. são frequentemente isoladas, e muitas destas infecções podem ser polimicrobianas (Jacobson 2007).

Para um diagnóstico definitivo de pneumonia bacteriana em répteis torna-se necessário uma abordagem clínica com exame físico, colheita de amostras apropriadas como lavado traqueal para cultura e a realização de exames ra- diológicos, que também podem ser úteis durante a avaliação de pacientes em resposta à terapia (Schumacher 2003, Murray 2006).0 presente relato tem por objetivo descrever o diagnóstico clínico, microbiológico, radiográfico e conduta terapêutica frente à pneumonia bacteriana em jabuti piranga (Chelonoidis carbonaria).

\section{MATERIAL E MÉTODOS}

Um jabuti-piranga (Chelonoidis carbonaria) fêmea, 30 anos, 10,1 $\mathrm{kg}$ foi atendido no Hospital Veterinário da Universidade Federal de Mato Grosso (Hovet-UFMT). A queixa do proprietário consistia no aparecimento de secreção muco purulenta nas narinas. Na anamnese foi descrito pneumonia diagnosticado radiologicamente há 6 meses, com prescrição de Enrofloxacina (5mg/kg/SID) comprimidos por 30 dias e suplemento de vitamina A 1000UI/kg. O proprietário relatou dificuldade na administração do antibiótico devido a sua forma de apresentação. A alimentação do animal não era adequada, consistindo em uma dieta composta de arroz, carne, macarrão e legumes, e o mesmo não se alimentava todos os dias. 0 jabuti era mantido em cativeiro em ambiente cimentado sem acesso à radiação solar. Ao exame físico constatou-se ferimento lacerante no membro posterior direito, presença de secreção mucosa na cavidade oral, dispneia, secreção muco purulenta nas narinas, epífora e diarreia.

0 animal foi encaminhado para o serviço de Diagnóstico por Imagem, onde foram realizadas radiografias na projeção dorsoventral e craniocaudal, onde o mesmo foi mantido em decúbito ventral durante o procedimento. Para a realização de exame radiográfico e lavado traqueal, o animal foi contido quimicamente com indução anestésica da associação de Cloridrato de Cetamina $(30 \mathrm{mg} / \mathrm{kg} / \mathrm{IM})$ e Midazolam $(2 \mathrm{mg} / \mathrm{kg} / \mathrm{IM})$, e mantido em máscara inalatória com sevoflurano.

Para obtenção de lavado traqueal para cultivo microbiológico e realização de antibiograma, o animal foi submetido à coleta quando ainda encontrava-se em plano anestésico. Este foi realizado com técnicas estéreis em um centro cirúrgico, adaptadas segundo Murray (2006). Após a identificação da glote do animal, uma sonda uretral número 8 estéril foi inserida pela traqueia até o pulmão. 0 comprimento de sonda introduzido foi baseado na sensibilidade tátil, até o início da resistência no momento da introdução da sonda. Com o auxílio de uma seringa de $20 \mathrm{~mL}$ acoplada na sonda uretral, foram inseridos $10 \mathrm{~mL}$ de solução fisiológica estéril ( $1 \mathrm{~mL}$ por kg de peso vivo do animal) e imediatamente aspirado, obtendo-se aproximadamente apenas $5 \mathrm{~mL}$ de volume resgatado. Em seguida, a extremidade distal da sonda foi dobrada e só então, a seringa desacoplada evitando a perda da pressão negativa, para se acoplar na mangueira de silicone do aspirador cirúrgico Inalamed $4 \mathrm{~L}^{\circledR} .0$ aspirador foi gentilmente acionado na potência mínima, visando a aspiração de qualquer resquício da solução fisiológica restante no pulmão.

0 material do lavado traqueal foi semeado em meios de cultivo Agar sangue (5\%), Agar MacConkey e Agar Sabouraud, incubadas à $37^{\circ} \mathrm{C}$ durante $48 \mathrm{~h}$ horas. A identificação e classificação bioquímica das colônias isoladas foi realizada segundo Quinn et al. 1994.

\section{RESULTADOS}

Os achados radiográficos foram sugestivos de pneumonia/edema pulmonar, pois foi observado consolidação do campo pulmonar esquerdo e um discreto aumento da opacidade no campo pulmonar direito. Baseado nos exames radiográficos e sinais clínicos apresentados iniciou-se o tratamento, enquanto foi aguardado o resultado micro- 
biológico. 0 tratamento consistiu de Cloranfenicol $40 \mathrm{mg} /$ $\mathrm{kg} / \mathrm{SID} / \mathrm{IM})$ e Furosemida (5mg/kg/SID/IM) por 10 dias, inalação com Acetilcisteína $10 \%$ (22mg/mL solução fisiológica/BID) pelo mesmo período de tempo e Vitamina A (1000UI $/ \mathrm{kg} / \mathrm{SID} / \mathrm{IM})$ por 7 dias, fluidoterapia utilizando Ringer Lactato $(10 \mathrm{~mL} / \mathrm{kg} / \mathrm{SID})$ via subcutânea dividida em duas aplicações e correção do manejo alimentar (Carpenter 2010).

Do material encaminhado para cultura foram isoladas duas bactérias pertencentes à Família Enterobacteriaceae, a Klebsiella spp. e Citrobacter spp. 0 teste de sensibilidade aos antimicrobianos foi realizado pelo método de difusão por discos (Bauer et al. 1966). Os antibióticos testados foram Gentamicina, Cloranfenicol, Enrofloxacina, Cefalexina, Estreptomicina, Amoxicilina, Ampicilina, Amoxilina e ácido clavulânico, e Sulfa/trimetropim, Ceftiofur, Doxiciclina e Claritromicina (Quadro 1).

A partir do resultado da cultura e antibiograma, novo protocolo antibiótico foi instituído com Gentamicina (5mg/ $\mathrm{kg} / \mathrm{IM}$ ) com sete aplicações, em intervalos de 72 horas, associado à fluidoterapia com Ringer lactato $(10 \mathrm{~mL} / \mathrm{kg} / \mathrm{SID})$ via subcutânea dividida em duas aplicações e inalação ultrassônica com Gentamicina (5mg/mL solução fisiológica/ BID), pelo período de 19 dias.

Novo exame radiográfico foi realizado após o tratamento, demonstrando uma discreta diminuição na opacidade do campo pulmonar direito e nenhuma alteração significativa no campo pulmonar esquerdo na projeção craniocaudal. Houve melhora dos sinais clínicos do animal, porém foi observada ainda a presença de secreção nasal.

Frente ao sinal clínico apresentado, nova coleta de material endotraqueal foi realizada, mantendo-se os protocolos anestésicos e protocolos microbiológicos anteriormente utilizados. Houve isolamento de Citrobacter spp. e Enterobacter spp. no segundo lavado endotraqueal. 0 antibiograma realizado mostrou sensibilidade dos micro-organismos a Amicacina (Quadro 2). A partir dos resultados obtidos, instituiu-se nova antibioticoterapia com uso de Amicacina $(2,5 \mathrm{mg} / \mathrm{kg} / \mathrm{IM})$, em sete aplicações com intervalos de $72 \mathrm{~h}$, pelo período de 19 dias.

Após antibioticoterapia, outro exame radiológico demonstrou redução satisfatória do quadro pulmonar e observou-se clinicamente desaparecimento de secreções nasais e epífora. Assim, a evolução do quadro após o terceiro protocolo antibiótico foi satisfatória quanto à redução dos sinais respiratórios, melhora clínica, e total aceitação do jabuti-piranga a nova dieta, incluindo vegetais folhosos, ovos e frutas. 0 animal foi liberado da internação com re- comendações ao proprietário de oferecer a dieta prescrita duas vezes ao dia, que fosse mantido em ambiente arejado com exposição diária à luz solar, e recolhido ao anoitecer para dentro do domicílio.

\section{DISCUSSÃO}

No presente relato foi diagnosticado um quadro de pneumonia em jabuti-piranga (Chelonoidis carbonaria), baseado em achados clínicos, microbiológicos e radiológicos, devido a provável ocorrência de uma associação entre deficiência nutricional, principalmente a hipovitaminose A e infecção bacteriana. Muitos testudinos tratados para pneumonia não respondem completamente ao tratamento, mesmo após a suplementação para pacientes com deficiência de vitamina A (Jacobson 2007).

As infecções respiratórias são comuns em répteis devido a diversos fatores, podendo ser fatal em testudinos. Os pulmões desses animais ocupam grande parte da cavidade interna e são compartimentados. Embora possua mucosa ciliada, os pulmões septados favorecem o acumulo de fluidos e dificultam expectoração. A ausência de diafragma verdadeiro faz com que estes animais não dependam da pressão torácica negativa para a respiração, sendo outro fator que dificulta a expectoração de secreções e corpos estranhos. (Frye 1991, Schumacher 2003, Stuart et al. 2004, O'Malley 2005, Cubas et al. 2006, Mader 2006, Lambertz et al. 2010, Bennett 2011). Outro fator que favorece infecções em testudinos pode ser associado à hipovitaminose $\mathrm{A}$, que leva à metaplasia escamosa e degeneração das superfícies epiteliais, incluindo os alvéolos pulmonares (Frye 1991, Stuart et al. 2004).

As bactérias mais frequentemente isoladas em répteis com pneumonia são Aeromonas spp, Edwarsiella tarda, Klebsiella spp, Pasteurella hemolytica, Pasteurella multocida, Pseudomonas aeruginosa, Staphylococcus aureus e Streptococcus spp. Bactérias como Bacterioides, Peptostreptococcus, Fusobacterium e Clostridium também são descritos. Há também o relato de bactérias atípicas como Mycoplasma e Chlamydia associadas à pneumonia em repteis (Frye 1991, Homer et al. 1994, Brown et al. 1999, Origgi \& Jacobson 2000, Mader 2006).

Pseudomonas aeruginosa foi isolada da cavidade oral e pulmões de serpentes tropicais (Boa constrictor e Pithon molurus), e verificou-se que todos os isolados foram sensíveis à gentamicina, tobramicina e Polimixina B (Aleksandrov \& Petkov 1985). Pneumonia bacteriana crônica foi diagnosticada em duas tartarugas com a identificação de bactérias como Pseudomonas spp. e membros da famí-

\section{Quadro 1. Resultados obtidos no teste de sensibilidade das bactérias identificadas a partir da primeira coleta do lavado traqueal do jabuti (Chelonoidis carbonaria)}

Bactérias isoladas GEN CLO ENR CFE EST AMO AMP AMC SUT CLA DOX CTF

\begin{tabular}{lllllllllllll}
\hline Klebsiella spp. & R & S & R & R & S & R & R & R & R & S & R & S \\
Citrobacter spp. & S & R & R & R & R & R & R & R & R & R & R & R
\end{tabular}

S = Sensível; R = Resistente; GEN = Gentamicina; $\mathrm{CLO}=$ Cloranfenicol; ENR = Enorfloxacina; $\mathrm{CFE}=$ Cefalexina; $\mathrm{EST}=$ Estreptomicina; $\mathrm{AMO}=$ Amoxicilina; $\mathrm{AMP}=$ Ampicilina; AMC = Amoxicilina e ácido clavulânico; SUT = Sulfa e trimetropim; CLA = Claritromicina; DOX = Doxiciclina; $\mathrm{CTF}=$ Ceftiofur. 


\section{Quadro 2. Resultados obtidos no teste de sensibilidade das bactérias identificadas a partir da segunda coleta do lavado traqueal do jabuti (Chelonoidis carbonaria)}

\begin{tabular}{|c|c|c|c|c|c|c|c|c|c|c|}
\hline Bactérias isoladas & GEN & $\mathrm{CLO}$ & CIP CFZ & CFL AMI & CTF PEN & $\mathrm{DOX}$ & AMP & AMO & EST & SUT \\
\hline Enterobacter spp. & $\mathrm{R}$ & $\mathrm{R}$ & $\mathrm{R}$ & $\mathrm{R}$ & $\mathrm{R}$ & $\mathrm{R}$ & $\mathrm{R}$ & $\mathrm{R}$ & $\mathrm{R}$ & $\mathrm{R}$ \\
\hline Citrobacter spp. & S & $\mathrm{R}$ & S & $\mathrm{R}$ & $\mathrm{R}$ & $\mathrm{R}$ & $\mathrm{S}$ & $\mathrm{S}$ & $\mathrm{S}$ & $\mathrm{R}$ \\
\hline
\end{tabular}

lia Enterobacteriaceae como Morganella morganii (Evans 1983). Citrobacter spp. foi o isolado dominante observado em estudo para verificação de bactérias resistentes a antibióticos em habitats marinhos a partir de liquido tubário da tartaruga verde (Chelonia mydas), seguido por Pseudomonas, Proteus, Enterobacter, Salmonella, Escherichia coli, Shigella, Edwardsiella, Morganella, Providencia e Arcomobacter, onde a maioria dos isolados foram resistentes à ampicilina (Al-Bahry et al. 2012).

As bactérias isoladas a partir do lavado traqueal do animal neste relato pertencem à família Enterobacteriaceae, sendo Klebsiella spp, especialmente Klebsiella pneumoniae comumente associadas com pneumonia em répteis (Mader 2006, Santoro et al. 2006). Citrobacter spp e Enterobacter spp não foram descritas associadas à pneumonia em jabutis, no entanto, estas fazem parte da microbiota oral e cloacal desses animais e outros repteis (McCoy \& Seidler 1973, Bagley \& Seidler 1977, Graevenitz 1977, Goldstein et al. 1981, Quinn et al. 1994, Jawetz et al. 1998, Santoro et al. 2006, Bastos et al. 2008, Al-Bahry et al. 2012, Benites et al. 2013).

Em seres humanos, Klebsiella pneumoniae está bem descrita como agente de infecções do trato respiratório, urinário e infecções hospitalares. A disseminação de determinantes de resistência como $\beta$-lactamases e carbapenemases entre agentes relacionados às infecções hospitalares vem emergindo, sendo mais comumente observados em membros da família Enterobacteriaceae, incluindo Klebsiella pneumoniae e Enterobacter cloacae (Graevenitz 1977, Moland et al. 2003, Luzzaro et al. 2004, Queenan \& Bush 2007, Zhengqing et al. 2014).

A criação dos jabutis como animais de estimação vem crescendo, e essa popularidade dos jabutis (Chelonoides spp.), tem sido motivo de preocupação, pois estes podem representar fonte potencial de infecção para seres humanos e animais, disseminando micro-organismos oportunistas e patogênicos no ambiente através das fezes (Benites et al. 2013). Essa espécie em vida livre alimenta-se de frutos, flores, cogumelos, sementes e menos frequentemente alimentam-se de insetos, vermes, material animal em putrefação e fezes. Os jovens consomem as fezes dos adultos para inocular a importante microbiota intestinal e auxiliar na digestão de material vegetal (Vogt 2008).

No presente relato foi constatada elevada resistência aos antibióticos testados. Em ambos os isolados obtidos das duas coletas de lavado traqueal, observou-se resistência para quase todas as classes de antimicrobianos testados na primeira e na segunda coleta, com perfil de resistência antimicrobiana diferente entre as mesmas, sendo todas com resistência múltipla (Quadro 1 e 2). 0 aparecimento de micro-organismos resistentes aos antibióticos vem crescendo, e segundo Kuiken et al. 2005, acredita-se que animais silvestres sejam a fonte de $70 \%$ das infecções emergentes. Em estudo realizado para identificar a susceptibilidade de bactérias aos antimicrobianos isoladas a partir de amostras de fezes, água e swab nasal provenientes de um zoológico no Japão observou-se a predominância de $E$. coli seguida de Klebsiella pneumoniae, e 21,1\% dos isolados foram resistentes à ampicilina, cefalotina, estreptomicina, sulfametoxazol-trimetoprim, canamicina, tetraciclina, ácido nalidíxico , e ciprofloxacina (Ahmed et al. 2007). Muitas penicilinas naturais, cefalosporinas de primeira geração e macrolídeos tem pouco efeito contra bactérias gram negativas, por fatores intrínsecos de resistência. Como a maioria dos isolados provenientes da pneumonia em répteis são bactérias gram negativas, recomenda-se a associação de antibióticos com amplo espectro de ação. Há sucesso na descrição sinérgica de beta lactâmicos e aminoglicosídeos. A septicemia pode ocorrer em casos mais graves ou prolongados de pneumonia em répteis (Mader 2006).

0 uso da fluidoterapia concomitantemente com o uso de drogas potencialmente nefrotóxicas é de suma importância, bem como o local de aplicação de tais antimicrobianos em virtude do sistema porta renal presente nos répteis. Dessa maneira, os antibióticos foram aplicados sempre nos grupos musculares dos membros anteriores. Além disso, considerando que o metabolismo dos répteis é mais lento que quando comparado com mamíferos e aves, a fluidoterapia auxilia no funcionamento renal (Jenkins 1996).

Em casos de pneumonia bacteriana em testudinos, o tratamento consiste em melhorias no manejo e antibioticoterapia sistêmica. A utilização da nebulização de antibióticos recomendados com solução fisiológica, posterior à nebulização contendo acetilcisteína pode favorecer a absorção do mesmo (Silvestre \& Massana 2008). A nebulização acrescida de antimicrobianos incluindo a amicacina tem sido utilizada em testudinos (Bennett 2011). Répteis com infecções respiratórias devem ser mantidos em conforto térmico para suas temperaturas preferenciais. $\mathrm{O}$ aumento da temperatura é importante não só para estimular o sistema imunológico, mas também para mobilizar secreções respiratórias (Jacobson 2007). De modo geral a temperatura de conforto para os testudinos está entre $25^{\circ} \mathrm{C} \mathrm{e} 30^{\circ} \mathrm{C}$, e mantê-los em cativeiro exige atenção a esses cuidados. Nessa espécie, temperaturas mais baixas e o stress podem desencadear queda do sistema imune e favorecer o aparecimento de infecções oportunistas (Cubas et al. 2006). 


\section{CONCLUSÕES}

No presente relato foi possível diagnosticar e tratar um espécime de jabuti piranga (Chelonoidis carbonaria) com pneumonia bacteriana e provável associação com a deficiência de vitamina A.

0 perfil de susceptibilidade dessas bactérias em relação aos antimicrobianos testados demonstrou a importância da realização do teste de antibiograma para estabelecer protocolo terapêutico eficaz, pois a administração empírica desses medicamentos pode provocar seleção de cepas resistentes.

\section{REFERÊNCIAS}

Ahmed A.M., Motoi Y., Sato M., Maruyama A., Watanabe H., Fukumoto Y. \& Shimamoto T. 2007. Zoo animals as reservoirs of gram-negative bacteria harboring integrons and antimicrobial resistence genes. Appl. Environ. Microbiol. 73:6686-6690.

Al-Bahry S.N., Al-Zadjali M.A., Mahmoud I.Y. \& Elshafie A.E. 2012. Biomonitoring marine habitats in reference to antibiotic resistant bacteria and ampicillin resistance determinants from oviductal fluid of the nesting green sea turtle, Chelonia mydas. Chemosphere 87:1308-1315.

Aleksandrov M. \& Petkov A. 1985. Case of Pseudomonas aeruginosa infection in tropical snakes. Vet. Med. Nauki 22:53-61.

Bauer A.W., Kirby W.M.M., Sheirris J.C. \& Turck M. 1966. Antibiotic susceptibility testingby a standardized single disk method. Am. J. Clin. Pathol. 45:493-496.

Bagley T.S. \& Seidler R.J. 1977. Significance of fecal coliform-positive Klebsiella. Appl. Environ. Microbiol. 33(5):1141-1148.

Bastos H.M., Lopes L.F.L., Gattamorta M.A. \& Matushima E.R. 2008. Prevalence of enterobacteria in Bothrops jararaca in São Paulo State: microbiological survey and antimicrobial resistance standards. Acta Scient. Biol. Sci. 30(3):321-326.

Bennett T. 2011. The Chelonian respiratory system. Vet. Clin. Exot. Anim. 14:225-239.

Benites N.R., Pessoa C., Bandini L., Saidenberg A., Moreno A., Sakata S., Gomes C. \& Melville P. 2013. Microbiota bacteriana e fúngica presentes na cloaca de jabutis-piranga (Geochelone carbonaria) criados em domicílio. Vet. Zootec. 20(1):102-110.

Brown M.B., McLaughlin G.S., Klein P.A., Crenshaw B.C., Schumacher I.M., Brown D.R. \& Jacobson E.R. 1999. Upper respiratory tract disease in the gopher tortoise is caused by Mycoplasma agassizii. J. Clin. Microbiol. 37:2262-2269.

Carpenter J.W. 2010. Formulário de Animais Exóticos. 3aㅡ ed. MedVet, São Paulo, 578p.

Cubas Z.S., Silva J.C.R. \& Catão-Dias J.L. 2006. Tratado de Animais Silvestres: Medicina Veterinaria. Roca, São Paulo, 1354p.

Evans R.H. 1983. Chronic bacterial pneumonia in free-ranging eastern Box turtles (Terrapene carolina carolina). J. Wildl. Dis. 19:349-352.

Frye F.L. 1991. Biomedical and surgical aspects of captive reptile husbandry. Vol.1. Krieger Publishing Company. Florida. 325p.

Graevenitz V.A. 1977. The role of opportunistic bacteria in human disease. Annu. Rev. Microbiol. 31:447-471.

Goldstein E.J.C., Agyare E.O., Vagvolgyi A.E. \& Halpern M. 1981. Aerobic bacterial oral flora of Garter snakes: development of normal flora and pathogenic potential for snakes and humans. J. Clin. Microbiol. 13(5):954-956.

Homer B.L., Jacobson E.R., Schumacher J. \& Scherba G. 1994. Chlamydiosis in Mariculture-reared Green Sea Turtles (Chelonia mydas). Vet. Pathol. 31:1-7.

Jacobson E. 2007. Infectious Diseases and Pathology of Reptiles: color atlas and text. Taylor and Francis Group. London, 716p.

Jenkins R.J. 1996. Diagnostic and Clinical Techniques, p.264-276. In: Mader D.R. (Ed.), Reptile Medicine and Surgery. 1aㅡ Ed. Saunders Elsevier, London, 512p.

Kuiken T., Leighton F.A., Fouchier R.A., LeDuc J.W., Peiris J.S., Schudel A., Stohr K. \& Osterhaus A.D. 2005. Public health: pathogen surveillance in animals. Science 309:1680-1681 (apud Ahmed et al. 2007).

Lambertz M., Böhme W. \& Perry S.F. 2010. The anatomy of the respiratory system in Platysternon megacephalum Gray, 1831 (Testudines: Cryptodira) and related species, and its phylogenetic implications. Comparative Biochemistry and Physiology, Part A. 156: 330-336.

Luzzaro F., Docquier J.D., Colinon C., Endimiani A., Lombardi G., Amicosante G., Rossolini G.M. \& Toniolo A. 2004. Emergence in Klebsiella pneumoniae and Enterobacter cloacae clinical isolates of the VIM-4 metallo$\beta$-lactamase encoded by a conjugative plasmid. Antimicrob. Agents Chemother. 48(2):648-650.

Mader D.R. 2006. Reptile Medicine and Surgery. $2^{\text {nd }}$ Ed. W.B. Saunders, London. $1242 \mathrm{p}$.

McCoy R.H. \& Seidler R.J. 1973. Potential pathogens in the environment: Isolation, enumeration, and identification of seven genera of intestinal bacteria associated with small green pet turtles. Appl. Microbiol. 25(4):534-538.

Moland E.S., Handson N.D., Herrera V.L., Black J.A., Lockhart T.J., Hossain A., Johnson J.A., Goering R.V. \& Thomson K.S. 2003. Plasmid-mediated, carbapenem-hydrolysing $\beta$-lactamase, KPC-2, in Klebsiella pneumoniae isolates. J. Antimicrob. Chemother. 51:711-714.

Murray J.M. 2006. Pneumonia and lower respiratory tract disease, p.865877. In Mader D.R. (Ed.), Reptile Medicine and Surgery. $2^{\text {nd }}$ ed. W.B. Saunders, London. 1242p.

O’Malley B. 2005. Clinical Anatomy and Physiology of Exotic Species. Elsevier Saunders, Philadelphia. 269p.

Origgi F.C. \& Jacobson E.R. 2000. Diseases of the respiratory tract of chelonians. Vet. Clin. North Am., Exot. Anim. Pract. 3:537-549.

Vogt R.C. 2008. Tartarugas da Amazônia. Gráfica Biblos, Lima.104p.

Queenan A.M. \& Bush K. 2007. Carbapenemases: the versatile $\beta$-lactamases. Clin. Microbiol. 20(3):440-458.

Quinn P.J., Carter M.E., Markey B.K. \& Carter G.R. 1994. Clinical Veterinary Microbiology. Mosby-Year Book Europe, London. 648p.

Santoro M., Hernandéz G., Caballero M. \& Garcia F. 2006. Aerobic bacterial flora of nesting green turtles (Chelonia mydas) from Tortuguero National Park, Costa Rica. J. Zoo Wildl. Med. 37(4):549-552.

Schumacher J. 2003. Reptile respiratory medicine. Vet. Clin. Exot. Anim. 6:213-231

Silvestre A.M. \& Massana J.S. 2008. Enfermedades Infecciosas y parasitarias en tortugas. Consulta. Difus. Vet. 150:43-54.

Stuart M., Wilkson R. \& Meyer J. 2004. Medicine and surgery of Tortoises and Turtles. Blackwell Publishing, Oxford, UK. 579p.

Zhengqing L., Yan Q., Xiang Q., Wei Y. \& Zeqing W. 2014. Emergence of Klebsiella pneumoniae carbapenemase-producing Escherichia coli sequence type 131 in Hangzhou, China. Chin. Med. J. 127(3):528-531. 